\title{
Imaging Techniques
}

\subsection{Association of Carotid Artery Stiffness Assessed with E-Tracking and}

Diastolic Dysfunction Assessed with Tissue Doppler Imaging Inpatients with

Masked Hypertension

F. Natale (1), C. Siniscalchi (1), L. Aronne (1), M. Credendino (1), R. Mocerino

(1), G. Tassinario (1), V. De Simone (1), L. Scotto Di Tella (1), S. Morra (1),

P. Calabrò (1), M.A. Tedesco (1), R. Calabrò (1)

(1)II Università di Napoli, Napoli, Italy

Introduction. Masked hypertension is defined as a normal blood pressure in the clinic or office, but an elevated blood pressure out of the clinic. It may occur in as many $10 \%$ of the general population. Increased arterial stiffness directly increase end-diastolic left ventricular pressure and compromise myocardial relaxation properties.

Aim. To evaluate the role of carotid artery stiffness parameters on diastolic function in patients with masked hypertension.

Methods. We have observed 70 consecutive patients with masked hypertension (proved by 24-hourambulatory blood pressure monitoring). Diastolic early (E') and late (A') velocities were recorded by pulse wave tissue Doppler imaging of the lateral mitral anulus in the apical 4-chamber view, furthermore carotid artery stiffness parameters were assessed by tracking on the left carotid with ALOKA_10 ultrasound system. Pulse wave velocity (PWV), elastic pressure (Ep) and stiffness parameter $(\beta)$ were measured.

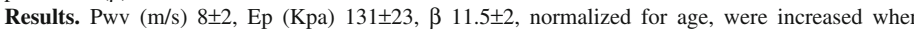
compared with healthy subjects. Stiffness parameters correlate significantly with Tissue Doppler imaging diastolic parameters in particularly with E' PWV $(r=0.66, p<0.05), E p(r=0.58, p<0.05)$ and $\beta(\mathrm{r}=0.67, \mathrm{p}<0.01)$

Conclusions. Arterial stiffness was increased in patients with masked hypertension and was associated with abnormal left ventricular diastolic function particularly with E', express ion of a greater contribution of atrial contraction to left ventricular filling for increased left ventricular end-diastolic pressure due to an high after-load and arterial stiffness. 